\title{
A limit formula for the quantum fidelity
}

\author{
Gaetana Spedalieri, ${ }^{1,}$ * Christian Weedbrook, ${ }^{2}$ and Stefano Pirandola ${ }^{1}$ \\ ${ }^{1}$ Department of Computer Science, University of York, York YO10 5GH, United Kingdom \\ ${ }^{2}$ Center for Quantum Information and Quantum Control, \\ Department of Electrical and Computer Engineering and Department of Physics, \\ University of Toronto, Toronto, M5S 3G4, Canada
}

(Dated: May 21, 2018)

\begin{abstract}
Quantum fidelity is a central tool in quantum information, quantifying how much two quantum states are similar. Here we propose a limit formula for the quantum fidelity between a mixed state and a pure state. As an example of an application, we apply this formula to the case of multimode Gaussian states, achieving a simple expression in terms of their first and second-order statistical moments.

PACS numbers: 03.67.--a, 03.65.--w, 42.50.-p
\end{abstract}

\section{INTRODUCTION}

Very often the performance of a quantum information protocol is measured in terms of similarity between two states. This happens both in dicrete (qubit) quantum information [1] and continuous-variable quantum information [2, 3], for instance, with Gaussian states [4, 5].

One of the most well known examples is that of quantum teleportation between two stations, where the perfect execution of the protocol corresponds to having an output state at the receiver's station which is equal to the input state originally processed at the sender's station [6 15]. Another important scenario is that of quantum cloning 16-20], where an unknown input quantum state is transformed into 2 or more clones. Because of the no-cloning theorem, the output clones cannot be identical to the input state [21, 22]. As a result, a measure of similarity between input state and output clones is fundamental in order to quantify the performance of a quantum cloning machine.

In these kinds of protocols, the typical measure of similarity between two quantum states is their fidelity. Quantum fidelity was introduced and characterized in two seminal papers by Uhlmann [23] and Jozsa 24]. Its general definition can be regarded as an extension of the wavefunction overlap to generally mixed quantum states. Despite its usefulness, simple closed formulas are not always easy to derive. For instance, we know a closed formula for the fidelity between two single-mode Gaussian states $25-$ 28], but a simple analytical result is still missing in the case of two arbitrary multimode Gaussian states.

Quantum fidelity also plays a central role in quantum hypothesis testing, where the basic problem is the discrimination between two equiprobable quantum states of a system by means of an optimal measurement. In this framework, quantum fidelity has been related to various other quantities such as the Helstrom bound 29 31] and the quantum Chernoff bound [32, 33], which provide a

*Electronic address: gae.spedalieri@york.ac.uk direct quantification of the minimum error probability affecting the state discrimination.

In this paper, we start from these connections to derive a new formula for the quantum fidelity between a generally mixed state and a pure state. This formula is expressed in the form of a limit and involves a generalized overlap between the two quantum states. This is the same kind of overlap which intervenes in the definition of the quantum Chernoff bound.

As an example of an application of this formula, we consider the bosonic setting and the case of multimode Gaussian states. Here we first introduce the notion of symplectic action, which enables us to simplify the symplectic manipulations of the second order moments. Then, by elaborating a result from Ref. 33], we derive a simple analytical expression for the fidelity between a mixed and a pure Gaussian state in terms of their first and second order moments.

The paper is organized as follows. In Sec. [II we provide a brief review of the basic facts regarding quantum fidelity and its connections with the various bounds used in quantum hypothesis testing. In Sec. III, we derive the limit formula for the quantum fidelity. Then, in Sec. IV, we consider bosonic continuous-variable systems. After a brief review of the basic notions on Gaussian states, we introduce the symplectic action and we derive the formula of the fidelity for Gaussian states. Finally, Sec. $\mathrm{V}$ is for conclusions.

\section{GENERAL NOTIONS ON QUANTUM FIDELITY}

Consider a quantum system with separable Hilbert space $\mathcal{H}$. In general, the states of this system are described by density operators $\rho: \mathcal{H} \rightarrow \mathcal{H}$ forming a corresponding state space $\mathcal{D}(\mathcal{H})$. Given two arbitrary states, $\rho$ and $\sigma$, their similarity can be quantified by the UhlmannJozsa fidelity [23, 24, 34]

$$
F(\rho, \sigma):=(\operatorname{Tr} \sqrt{\sqrt{\rho} \sigma \sqrt{\rho}})^{2} .
$$


This is a positive number $0 \leq F \leq 1$, where $F=1$ corresponds to identical states, and $F=0$ corresponds to orthogonal states, i.e., density operators with orthogonal supports in $\mathcal{D}(\mathcal{H})$. In the case where one of the states is pure $\sigma=|\varphi\rangle\langle\varphi|$, the fidelity assumes the sandwich expression

$$
F(\rho,|\varphi\rangle)=\langle\varphi|\rho| \varphi\rangle
$$

which becomes the overlap

$$
F(|\psi\rangle,|\varphi\rangle)=|\langle\psi \mid \varphi\rangle|^{2},
$$

if also the other state is pure $\rho=|\psi\rangle\langle\psi|$.

Most of the properties of the quantum fidelity can be derived from Uhlmann's theorem which states that

$$
F(\rho, \sigma)=\max _{\left|\varphi_{\sigma}\right\rangle}\left|\left\langle\varphi_{\rho} \mid \varphi_{\sigma}\right\rangle\right|^{2},
$$

where $\left|\varphi_{\rho}\right\rangle$ and $\left|\varphi_{\sigma}\right\rangle$ are purifications of $\rho$ and $\sigma$, respectively. For instance, immediate consequences of this theorem are the positive range $0 \leq F \leq 1$, the symmetry property $F(\rho, \sigma)=F(\sigma, \rho)$, and the invariance $F(\rho, \sigma)=F\left(U \rho U^{\dagger}, U \sigma U^{\dagger}\right)$ under a generic unitary $U$.

Despite being a measure of similarity between two quantum states, the quantum fidelity is not properly a metric in the state space $\mathcal{D}(\mathcal{H})$. In fact, by definition, a metric in $\mathcal{D}(\mathcal{H})$ is a map $D:(\rho, \sigma) \rightarrow \mathbb{R}$ with the following properties:

(i) Positive definiteness, i.e., $D(\rho, \sigma) \geq 0(=0 \Leftrightarrow \sigma=\rho)$;

(ii) Symmetry, i.e., $D(\rho, \sigma)=D(\sigma, \rho)$;

(iii) Subadditivity or triangle inequality, i.e., $D(\rho, \gamma) \leq$ $D(\rho, \sigma)+D(\sigma, \gamma)$, for any triplet $\rho, \sigma$ and $\gamma$.

In this list, the fidelity fails both the first property (since $F(\rho, \rho)=1)$ and the subadditivity.

Even if it is not a metric by itself, we can easily connect the quantum fidelity to a metric in $\mathcal{D}(\mathcal{H})$. For instance, we can consider the Bures' distance [31]

$$
D_{B}(\rho, \sigma)=\sqrt{2-2 \sqrt{F(\rho, \sigma)}},
$$

or the angular distance [1]

$$
D_{A}(\rho, \sigma)=\operatorname{Arccos} \sqrt{F(\rho, \sigma)} .
$$

Most importantly, the quantum fidelity can be connected with the trace distance, which is the standard metric adopted in quantum information for its direct interpretation in quantum hypothesis testing.

Given two quantum states, $\rho$ and $\sigma$, their trace distance is defined as [1, 35]

$$
D(\rho, \sigma)=\frac{1}{2}\|\rho-\sigma\|_{1},
$$

where

$$
\|O\|_{1}:=\operatorname{Tr}|O|=\operatorname{Tr} \sqrt{O^{\dagger} O}
$$

is the trace norm of an arbitrary trace-class operator $O$ [36]. The trace distance ranges in the positive interval $[0,1]$, with $D=0$ for identical states and $D=1$ for orthogonal states. $D(\rho, \sigma)$ determines the error probability which affects the discrimination of the two states, $\rho$ and $\sigma$, by means of an optimal quantum measurement. Suppose that a system is prepared in one of two equiprobable states, $\rho$ and $\sigma$, then the optimal positive operator valued measure (POVM) provides the correct answer with an error probability given by the Helstrom bound [29]

$$
P_{\text {err }}=\frac{1-D}{2} .
$$

According to Ref. [30], we can use the trace distance to write the following upper bound for the fidelity

$$
F \leq 1-D^{2} .
$$

Then, according to Ref. [1] we can also write the lower bound

$$
(1-D)^{2} \leq F
$$

In particular, if one of the states is pure $\sigma=|\varphi\rangle\langle\varphi|$ we have the tighter lower bound [1]

$$
1-D \leq F .
$$

Finally if both the states are pure, $\rho=|\psi\rangle\langle\psi|$ and $\sigma=$ $|\varphi\rangle\langle\varphi|$, then we have the equality [29]

$$
F=1-D^{2} .
$$

Besides the trace distance and the Helstrom bound, the quantum fidelity possesses important relations with other crucial quantities in quantum hypothesis testing: the quantum Chernoff bound [32] and the quantum Battacharyya bound [33]. Let us consider the quantity

$$
C_{s}(\rho, \sigma):=\operatorname{Tr}\left(\rho^{s} \sigma^{1-s}\right) \leq 1,
$$

which represents a generalized $s$-overlap between the two states $\rho$ and $\sigma$. Using Eq. (14), we can define the Chernoff term

$$
C(\rho, \sigma):=\inf _{s \in(0,1)} C_{s}(\rho, \sigma),
$$

and the Battacharyya term

$$
B(\rho, \sigma):=C_{1 / 2}(\rho, \sigma)=\operatorname{Tr} \sqrt{\rho} \sqrt{\sigma} .
$$

Up to a factor 2, these terms provide the (single-shot) formulae for the quantum Chernoff and Battacharrya bounds, which are used to estimate the minimum error probability in the discrimination of $\rho$ and $\sigma$ via a single quantum measurement, i.e., we have

$$
P_{\text {err }} \leq \frac{C}{2}, P_{\text {err }} \leq \frac{B}{2} .
$$


It is straightforward to prove the following chain of inequalities involving the fidelity

$$
C \leq B \leq \sqrt{F}
$$

In fact $C \leq B$ is trivial, while $B \leq \sqrt{F}$ comes from the fact that 24,30 .

$$
\operatorname{Tr} \sqrt{\rho} \sqrt{\sigma}=|\operatorname{Tr} \sqrt{\rho} \sqrt{\sigma}| \leq \operatorname{Tr}|\sqrt{\rho} \sqrt{\sigma}|=\operatorname{Tr} \sqrt{\sqrt{\rho} \sigma \sqrt{\rho}}
$$

where we exploit the inequality $|\operatorname{Tr} O| \leq \operatorname{Tr}|O|$ valid for any trace-class operator $O$.

\section{QUANTUM FIDELITY BETWEEN A PURE STATE AND A MIXED STATE}

In this section we focus our attention to the case of a mixed state $\rho$ and a pure state $\sigma=|\varphi\rangle\langle\varphi|$. In this specific case, we prove that the fidelity can be simply expressed as a limit formula involving the $s$-overlap.

Before stating this result, it is important to note that the Chernoff term of Eq. (15) is defined in terms of an infimum over the open interval $(0,1)$. In fact, despite the $s$-overlap $C_{s}(\rho, \sigma) \leq 1$ is correctly defined for any $s$ in the closed interval $[0,1]$, the two border points $s=0$ and $s=1$ can be excluded from its minimization, since we always have

$$
C_{0}=\operatorname{Tr}|\varphi\rangle\langle\varphi|=1, C_{1}=\operatorname{Tr} \rho=1 .
$$

Besides the restriction of the interval $[0,1] \rightarrow(0,1)$, it is also essential to consider an infimum instead of a minimum in Eq. (15). In fact, there are nontrivial situations where a minimum does not exist and an infimum is defined in the limit of $s \rightarrow 0^{+}$or $s \rightarrow 1^{-}$. This is exactly what happens when one of the two states is pure. In this case the $s$-overlap $C_{s}$ tends to the quantum fidelity, which becomes equal to the Chernoff term. These are the main contents of the following results.

Theorem 1 Given a mixed state $\rho$ and a pure state $|\varphi\rangle\langle\varphi|$, their quantum fidelity can be expressed as

$$
F(\rho,|\varphi\rangle)=\lim _{s \rightarrow 1^{-}} C_{s}(\rho,|\varphi\rangle)=\lim _{s \rightarrow 1^{-}} \operatorname{Tr}\left(\rho^{s}|\varphi\rangle\left\langle\left.\varphi\right|^{1-s}\right) .\right.
$$

Proof. Specify the definition of Eq. (14) to the case where $\sigma=|\varphi\rangle\langle\varphi|$, i.e.,

$$
C_{s}=\operatorname{Tr}\left(\rho^{s}|\varphi\rangle\left\langle\left.\varphi\right|^{1-s}\right)\right.
$$

For every $s \in(0,1)$ we can use the property of the projector

$$
|\varphi\rangle\left\langle\left.\varphi\right|^{1-s}=\mid \varphi\right\rangle\langle\varphi|
$$

and write

$$
C_{s}=\left\langle\varphi\left|\rho^{s}\right| \varphi\right\rangle
$$

Now, we can always decompose $\rho$ as

$$
\rho=\sum_{k} p_{k}^{1 / 2}|k\rangle\langle k|,
$$

where $p_{k} \in[0,1]$ for any $k$, and $\{|k\rangle\}$ is an orthonormal set (this is just the spectral decomposition of the state).

Taking the $s$-power of Eq. (24), we get

$$
\rho^{s}=\sum_{k} p_{k}^{s / 2}|k\rangle\langle k|,
$$

for any $s \in(0,1)$. Using the latter equation in Eq. (23), we achieve

$$
C_{s}=\sum_{k} p_{k}^{s / 2}|\langle k \mid \varphi\rangle|^{2} .
$$

Finally, taking the limit of $s \rightarrow 1^{-}$, we derive

$$
\begin{aligned}
\lim _{s \rightarrow 1^{-}} C_{s} & =\sum_{k} p_{k}^{1 / 2}|\langle k \mid \varphi\rangle|^{2} \\
& =\langle\varphi|\rho| \varphi\rangle=F(\rho,|\varphi\rangle),
\end{aligned}
$$

which corresponds to the result of Eq. (20).

Note that we can equivalently write

$$
F(|\varphi\rangle, \rho)=\lim _{s \rightarrow 0^{+}} C_{s}(|\varphi\rangle, \rho)=\lim _{s \rightarrow 0^{+}} \operatorname{Tr}\left(|\varphi\rangle\left\langle\left.\varphi\right|^{s} \rho^{1-s}\right) .\right.
$$

As an application of the previous theorem, we have the following corollary, which is a result already known in the literature (e.g., see Refs. [32, 37]).

Corollary 2 Given a mixed state $\rho$ and a pure state $|\varphi\rangle\langle\varphi|$, their quantum fidelity can be expressed as

$$
F(\rho,|\varphi\rangle)=C(\rho,|\varphi\rangle) .
$$

Proof. This is a trivial consequence of the previous theorem. In fact, we have that $C_{s}$ in Eq. (26) is manifestly non-increasing in $s$. As a consequence, we have

$$
C=\inf _{s \in(0,1)} C_{s}=\lim _{s \rightarrow 1^{-}} C_{s}=F(\rho,|\varphi\rangle),
$$

which completes the proof.

The limit formula of Theorem 1 is useful in all those scenarios where the $s$-overlap $C_{s}$ is easy to compute. For instance, one of these scenarios is that of Gaussian states. As we show in the next section, we can derive a very simple formula for the fidelity between a pure and a mixed Gaussian state in terms of their statistical moments.

\section{FORMULA FOR GAUSSIAN STATES}

In this section we apply the limit formula to the case of multimode Gaussian states. We first review some basic facts about bosonic systems, symplectic algebra and Gaussian states. Then, we introduce the notion of symplectic action, that we use to re-formulate the expression of the $s$-overlap between two arbitrary Gaussian states. From this expression, we finally derive the formula for the fidelity between two multimode Gaussian states, in the case where one of the two states is pure. 


\section{A. Basic notions about Gaussian states}

Let us consider a bosonic system of $n$ modes. This quantum system is described by a tensor product Hilbert space $\mathcal{H}^{\otimes n}$ and a vector of quadrature operators

$$
\hat{\mathbf{x}}^{T}:=\left(\hat{q}_{1}, \hat{p}_{1}, \ldots, \hat{q}_{n}, \hat{p}_{n}\right)
$$

satisfying the commutation relations 38]

$$
\left[\hat{\mathbf{x}}, \hat{\mathbf{x}}^{T}\right]=2 i \Omega
$$

where

$$
\boldsymbol{\Omega}:=\bigoplus_{i=1}^{n}\left(\begin{array}{cc}
0 & 1 \\
-1 & 0
\end{array}\right)
$$

The matrix of Eq. (33) defines a symplectic form in $\mathbb{R}^{2 n}$. Correspondingly, a real matrix $\mathbf{S}$ is called "symplectic" when it preserves $\boldsymbol{\Omega}$ by congruence, i.e.,

$$
\mathbf{S} \Omega \mathbf{S}^{T}=\boldsymbol{\Omega}
$$

By definition a quantum state $\rho$ of a bosonic system is called "Gaussian" when its phase-space representation is Gaussian [4]. In such a case, the quantum state is completely described by the first two statistical moments. Thus, a Gaussian state $\rho$ of $n$ bosonic modes is characterized by a displacement vector

$$
\overline{\mathbf{x}}:=\operatorname{Tr}(\hat{\mathbf{x}} \rho)
$$

and a covariance matrix $(\mathrm{CM})$

$$
\mathbf{V}:=\frac{1}{2} \operatorname{Tr}\left(\left\{\hat{\mathbf{x}}, \hat{\mathbf{x}}^{T}\right\} \rho\right)-\overline{\mathbf{x}} \overline{\mathbf{x}}^{T}
$$

where $\{$,$\} denotes the anticommutator [38]. According$ to the definition, a $\mathrm{CM}$ is a $2 n \times 2 n$ real and symmetric matrix. Furthermore, it must satisfy the uncertainty principle 39]

$$
\mathbf{V}+i \boldsymbol{\Omega} \geq 0
$$

A Gaussian state is pure if and only if its CM has unit determinant. In fact, one can easily prove that

$$
\operatorname{Tr} \rho^{2}=\frac{1}{\sqrt{\operatorname{det} \mathbf{V}}},
$$

for a Gaussian state.

\section{B. Symplectic action}

An important tool in the study of Gaussian states is Williamson's theorem [40], which assures the symplectic decomposition of a generic CM. In fact, for every CM V, there exists a symplectic matrix $\mathbf{S}$ such that

$$
\mathbf{V}=\mathbf{S W S}^{T}
$$

where

$$
\mathbf{W}=\bigoplus_{i=1}^{n} \nu_{i} \mathbf{I}, \quad \mathbf{I}:=\left(\begin{array}{ll}
1 & \\
& 1
\end{array}\right) .
$$

The matrix $\mathbf{W}$ is called the "Williamson form" of $\mathbf{V}$, and the set $\left\{\nu_{i}\right\}=\left\{\nu_{1}, \cdots, \nu_{n}\right\}$ is called the "symplectic spectrum" of V. As a consequence of the uncertainty principle, each symplectic eigenvalue $\nu_{i}$ must be greater than or equal to the quantum shot-noise (here corresponding to 1). More exactly, the uncertainty principle of Eq. (37) is equivalent to the conditions [4, 41]

$$
\left.\mathbf{V}>0, \nu_{i} \geq 1 \text { (for any } i\right) \text {. }
$$

In particular, a Gaussian state is pure if and only if its symplectic spectrum is all equal to one $\left(\nu_{i}=1\right.$ for any $\left.i\right)$. In other words, for a pure Gaussian state, the Williamson form is equal to the identity. This is a direct consequence of Eqs. (38) and (41) plus the fact that the determinant is a global symplectic invariant (i.e., $\operatorname{det} \mathbf{V}=\operatorname{det} \mathbf{W}$ ).

Now, consider a real function $f: \mathbb{R} \rightarrow \mathbb{R}$ and generic CM V with symplectic decomposition

$$
\mathbf{V}=\mathbf{S}\left[\bigoplus_{i=1}^{n} \nu_{i} \mathbf{I}\right] \mathbf{S}^{T}
$$

Then, we define the "symplectic action" $f(\mathbf{V})_{*}$ of $f$ over $\mathbf{V}$ the following matrix

$$
f(\mathbf{V})_{*}=\mathbf{S}\left[\bigoplus_{i=1}^{n} f\left(\nu_{i}\right) \mathbf{I}\right] \mathbf{S}^{T}
$$

Since the symplectic decomposition is unique (up to uninfluential local rotations), the output matrix $f(\mathbf{V})_{*}$ is unambiguously defined. In particular, this matrix is a CM if and only if $f\left(\nu_{i}\right) \geq 1$ for every $i$. It is also clear that $f\left(\mathbf{S V S}^{T}\right)_{*}=\mathbf{S} f(\mathbf{V})_{*} \mathbf{S}^{T}$ for every CM V and symplectic matrix $\mathbf{S}$.

It is important to note that this operation is different from the standard notion of function of a matrix $f(\mathbf{V})$, where $f$ is applied to the standard eigenvalues of the spectral decomposition of $\mathbf{V}$. We have $f(\mathbf{V})_{*}=f(\mathbf{V})$ only if spectral and symplectic decompositions coincide, which happens when the symplectic matrix $\mathbf{S}$ is a proper rotation (so that $\mathbf{S}^{T}=\mathbf{S}^{-1}$ ). In general, the symplectic action is a useful tool which enables us to simplify the formalism in the manipulation of the CMs.

\section{From the $s$-overlap to the quantum fidelity}

According to Ref. [33], we can write a closed formula for the $s$-overlap between two arbitrary multimode Gaussian states. Here we briefly review this formula by adopting the formalism of the symplectic action.

First of all, let us define the two real functions

$$
G_{p}(x):=\frac{2^{p}}{(x+1)^{p}-(x-1)^{p}},
$$


and

$$
\Lambda_{p}(x):=\frac{(x+1)^{p}+(x-1)^{p}}{(x+1)^{p}-(x-1)^{p}}
$$

which are finite and non-negative for every $x \geq 1$ and $p>0$. Using these functions, we can easily express the $s$-overlap between two arbitrary $n$-mode Gaussian states, $\rho_{0}$ and $\rho_{1}$, with statistical moments $\left\{\overline{\mathbf{x}}_{0}, \mathbf{V}_{0}\right\}$ and $\left\{\overline{\mathbf{x}}_{1}, \mathbf{V}_{1}\right\}$, and associated symplectic spectra $\left\{\nu_{i}^{0}\right\}$ and $\left\{\nu_{i}^{1}\right\}$. In fact, for any $0<s<1$, their $s$-overlap is given by

$$
C_{s}\left(\rho_{0}, \rho_{1}\right)=\Pi_{s}\left(\operatorname{det} \Sigma_{s}\right)^{-1 / 2} \exp \left(-\frac{\mathbf{d}^{T} \Sigma_{s}^{-1} \mathbf{d}}{2}\right),
$$

where $\mathbf{d}:=\overline{\mathbf{x}}_{0}-\overline{\mathbf{x}}_{1}$

$$
\Sigma_{s}:=\Lambda_{s}\left(\mathbf{V}_{0}\right)_{*}+\Lambda_{1-s}\left(\mathbf{V}_{1}\right)_{*},
$$

and

$$
\Pi_{s}:=2^{n} \prod_{i=1}^{n} G_{s}\left(\nu_{i}^{0}\right) G_{1-s}\left(\nu_{i}^{1}\right) .
$$

Note that the symplectic action intervenes in Eq. (47). Explicitly, we have

$$
\begin{aligned}
\mathbf{V}_{0} & =\mathbf{S}_{0}\left[\bigoplus_{i=1}^{n} \nu_{i}^{0} \mathbf{I}\right] \mathbf{S}_{0}^{T} \\
& \rightarrow \Lambda_{s}\left(\mathbf{V}_{0}\right)_{*}=\mathbf{S}_{0}\left[\bigoplus_{i=1}^{n} \Lambda_{s}\left(\nu_{i}^{0}\right) \mathbf{I}\right] \mathbf{S}_{0}^{T},
\end{aligned}
$$

and

$$
\begin{aligned}
\mathbf{V}_{1} & =\mathbf{S}_{1}\left[\bigoplus_{i=1}^{n} \nu_{i}^{1} \mathbf{I}\right] \mathbf{S}_{1}^{T} \\
& \rightarrow \Lambda_{1-s}\left(\mathbf{V}_{1}\right)_{*}=\mathbf{S}_{1}\left[\bigoplus_{i=1}^{n} \Lambda_{1-s}\left(\nu_{i}^{1}\right) \mathbf{I}\right] \mathbf{S}_{1}^{T}
\end{aligned}
$$

The formula of the $s$-overlap can be greatly simplified in the presence of pure Gaussian states, on which the two functions $\Lambda_{p}$ and $G_{p}$ have a trivial action. In fact, suppose that a Gaussian state $\rho$ is pure. This means that its symplectic spectrum is all equal to one, i.e., $\nu_{i}=1$ for any $i$. In other words, its CM has symplectic decomposition

$$
\mathbf{V}=\mathbf{S}\left[\bigoplus_{i=1}^{n} \mathbf{I}\right] \mathbf{S}^{T}
$$

where the Williamson form corresponds to the $n$-mode identity matrix. Then, for every $p>0$, we have

$$
\Lambda_{p}(\mathbf{V})_{*}=\mathbf{V},
$$

i.e., the symplectic action of $\Lambda_{p}$ does not change pure CMs. In fact, explicitly we have

$$
\Lambda_{p}(\mathbf{V})_{*}=\mathbf{S}\left[\bigoplus_{i=1}^{n} \Lambda_{p}(1) \mathbf{I}\right] \mathbf{S}^{T}=\mathbf{S}\left[\bigoplus_{i=1}^{n} \mathbf{I}\right] \mathbf{S}^{T}=\mathbf{V}
$$

where we use the fact that $\Lambda_{p}(1)=1$ for any $p>0$. Also the computation of $G_{p}$ becomes trivial. In fact, for any $p>0$ we have

$$
G_{p}\left(\nu_{i}\right)=G_{p}(1)=1 .
$$

Coming back to the formula of Eq. (46), if one of the two Gaussian state is pure, e.g., $\rho_{1}=\left|\varphi_{1}\right\rangle\left\langle\varphi_{1}\right|$, then we have the simplifications

$$
\Pi_{s}=2^{n} \prod_{i=1}^{n} G_{s}\left(\nu_{i}^{0}\right)
$$

and

$$
\Sigma_{s}=\Lambda_{s}\left(\mathbf{V}_{0}\right)_{*}+\mathbf{V}_{1},
$$

for every $s \in(0,1)$. Now, by taking the limit of $s \rightarrow 1^{-}$, we can derive the formula for Gaussian states.

Theorem 3 Let us consider two n-mode Gaussian states, $\rho_{0}$ and $\rho_{1}$, where $\rho_{0}$ is generally mixed (with moments $\overline{\mathbf{x}}_{0}$ and $\mathbf{V}_{0}$ ) and $\rho_{1}=\left|\varphi_{1}\right\rangle\left\langle\varphi_{1}\right|$ is pure (with moments $\overline{\mathbf{x}}_{1}$ and $\left.\mathbf{V}_{1}\right)$. Their fidelity $F=F\left(\rho_{0},\left|\varphi_{1}\right\rangle\right)$ can be computed via the formula

$$
F=\frac{2^{n}}{\sqrt{\operatorname{det}\left(\mathbf{V}_{0}+\mathbf{V}_{1}\right)}} \exp \left[-\frac{\mathbf{d}^{T}\left(\mathbf{V}_{0}+\mathbf{V}_{1}\right)^{-1} \mathbf{d}}{2}\right]
$$

where $\mathbf{d}:=\overline{\mathbf{x}}_{0}-\overline{\mathbf{x}}_{1}$.

Proof. This proof simply combines the limit formula of the fidelity, given in Eq. (20), with the analytical formula of $C_{s}$ for Gaussian states. Given two Gaussian states $\rho_{0}$ and $\rho_{1}=\left|\varphi_{1}\right\rangle\left\langle\varphi_{1}\right|$, their $s$-overlap $C_{s}\left(\rho_{0},\left|\varphi_{1}\right\rangle\right)$ is expressed by Eq. (46) with the simplified terms of Eqs. (55) and (56), where $\left\{\nu_{i}^{0}\right\}$ is the symplectic spectrum of $\rho_{0}$. Now taking the limit of $s \rightarrow 1^{-}$in $C_{s}\left(\rho_{0},\left|\varphi_{1}\right\rangle\right)$ is equivalent to taking the limit of $s \rightarrow 1^{-}$in the terms $\Pi_{s}$ and $\Sigma_{s}$ of Eqs. (55) and (56).

Since $G_{s}$ and $\Lambda_{s}$ are continuous at $s=1$, we have

$$
\begin{gathered}
\lim _{s \rightarrow 1^{-}} G_{s}(x)=G_{1}(x)=1, \\
\lim _{s \rightarrow 1^{-}} \Lambda_{s}(x)=\Lambda_{1}(x)=x,
\end{gathered}
$$

for every $x \geq 1$. In particular, for every CM $\mathbf{V}$, we can write

$$
\lim _{s \rightarrow 1^{-}} \Lambda_{s}(\mathbf{V})_{*}=\Lambda_{1}(\mathbf{V})_{*}=\mathbf{V} .
$$

By applying these properties to the state $\rho_{0}$, we get

$$
\lim _{s \rightarrow 1^{-}} G_{s}\left(\nu_{i}^{0}\right)=1, \lim _{s \rightarrow 1^{-}} \Lambda_{s}\left(\mathbf{V}_{0}\right)_{*}=\mathbf{V}_{0} .
$$


As a consequence, we can write

$$
\lim _{s \rightarrow 1^{-}} \Pi_{s}=2^{n}, \lim _{s \rightarrow 1^{-}} \Sigma_{s}=\mathbf{V}_{0}+\mathbf{V}_{1} .
$$

Now, using Eq. (62) and Eq. (46), we get

$$
\lim _{s \rightarrow 1^{-}} C_{s}\left(\rho_{0},\left|\varphi_{1}\right\rangle\right)=\frac{2^{n}}{\sqrt{\operatorname{det}\left(\mathbf{V}_{0}+\mathbf{V}_{1}\right)}} e^{-\frac{\mathbf{d}^{T}\left(\mathbf{V}_{0}+\mathbf{V}_{1}\right)^{-1} \mathbf{d}}{2}},
$$

which provides the result of the theorem.

\section{CONCLUSION}

In conclusion, we have provided a limit formula for computing the quantum fidelity between a mixed and a pure state. This formula involves a generalized $s$-overlap between the two quantum states, a quantity used in the definition of the quantum Chernoff bound. As an application of the formula, we have considered the case of Gaussian states, for which we have derived a simple expression in terms of their first and second-order statistical moments.
An alternative formula for the computation of the quantum fidelity can be useful in many scenarios, including protocols of quantum teleportation [6-15], entanglement swapping [42 44], and quantum cloning [1620]. Clearly, other important areas of application are quantum state discrimination (i.e., quantum hypothesis testing) and quantum channel discrimination, where the latter includes practical problems such as the quantum illumination of targets [45, 46] and the quantum reading of classical digital memories [47 53].

\section{Acknowledgments}

C.W. acknowledges support from the Ontario postdoctoral fellowship program, the CQIQC postdoctoral fellowship program, CIFAR, the Canada Research Chair program, NSERC, QuantumWorks. S. P. acknowledges support from EPSRC (Grant No. EP/J00796X/1). Authors would like to thank Aharon Brodutch for spotting a previous error in the proof of Theorem 1.
[1] M. A. Nielsen and I. L. Chuang, Quantum Computation and Quantum Information (Cambridge University Press, Cambridge, 2000).

[2] S. L. Braunstein and A. K. Pati, Quantum Information Theory with Continuous Variables, (Kluwer Academic, Dordrecht, 2003).

[3] S. L. Braunstein and P. van Loock, Rev. Mod. Phys. 77, 513 (2005).

[4] C. Weedbrook, S. Pirandola, R. Garcia-Patron, N. J. Cerf, T. C. Ralph, J. H. Shapiro, and S. Lloyd, preprint arXiv:1110.3234 (2011). To appear on RMP.

[5] A. Ferraro, S. Olivares, and M. Paris, Gaussian states in quantum information, ISBN 88-7088-483-X (Biliopolis, Napoli, 2005).

[6] A. Furusawa et al., Science 282, 706 (1998).

[7] S. L. Braunstein, and H. J. Kimble, Phys. Rev. Lett. 80, 869 (1998).

[8] T. C. Ralph, Optics Letters 24, 348 (1999).

[9] P. van Loock, and S. L. Braunstein, Phys. Rev. Lett. 84, 3482 (2000).

[10] S. Pirandola, S. Mancini, D. Vitali, and P. Tombesi, Phys. Rev. A 68, 062317 (2003).

[11] S. D. Bartlett and W. J. Munro, Phys. Rev. Lett. 90, 117901 (2003).

[12] S. Pirandola, Int. J. Quant. Inf. 3, 239 (2005).

[13] S. Pirandola, S. Mancini, and D. Vitali, Phys. Rev. A 71, 042326 (2005).

[14] S. Pirandola and S. Mancini, Laser Physics 16, 1418 (2006).

[15] J. Sherson, H. Krauter, R. K. Olsson, B. Julsgaard, K. Hammerer, I. Cirac, and E. S. Polzik, Nature 443, 557 (2009).

[16] V. Bužek and M. Hillery, Phys. Rev. A 54, 1844 (1996).

[17] G. Lindblad, J. Phys. A 33, 5059 (2000)
[18] N. J. Cerf, A. Ipe, and X. Rottenberg, Phys. Rev. Lett. 85, 1754 (2000).

[19] S. L. Braunstein, N. J. Cerf, S. Iblisdir, P. van Loock, and S. Massar, Phys. Rev. Lett. 86, 4938 (2001).

[20] J. Fiuràšek, Phys. Rev. Lett. 86, 4942 (2001).

[21] W. K. Wootters and W. H. Zurek, Nature 299, 802 (1982).

[22] D. Dieks, Phys. Lett. 92A, 271 (1982).

[23] A. Uhlmann, Rep. Math. Phys. 9, 273 (1976).

[24] R. Jozsa, Journal of Modern Optics 41, 2315 (1994).

[25] A. S. Holevo, IEEE Trans. Inform. Theory 21, 533 (1975).

[26] H. Nha, and H. J. Carmichael, Phys. Rev. A 71, 032336 (2005).

[27] S. Olivares, M. G. A. Paris, and U. L. Andersen, Phys. Rev. A 73, 062330 (2006).

[28] H. Scutaru, J. Phys. A 31, 3659 (1998).

[29] C. W. Helstrom, Quantum detection and estimation theory (Academic Press, New York, 1976).

[30] C. A. Fuchs and J. V. de Graaf, IEEE Trans. Inf. Theory 45, 1216 (1999).

[31] C. Fuchs, PhD thesis (Univ. of New Mexico, Albuquerque, 1995).

[32] K. M. R. Audenaert et al., Phys. Rev. Lett. 98, 160501 (2007); J. Calsamiglia et al., Phys. Rev. A 77, 032311 (2008).

[33] S. Pirandola and S. Lloyd, Phys. Rev. A 78, 012331 (2008).

[34] An alternative definition which is commonly adopted in quantum information is the square-root or Bures' fidelity. This corresponds to the square root of the UhlmannJozsa fidelity

$$
F_{B}(\rho, \sigma):=\sqrt{F(\rho, \sigma)}=\operatorname{Tr} \sqrt{\sqrt{\rho} \sigma \sqrt{\rho}} .
$$


Bures' fidelity is the direct generalization of the classical fidelity. In fact, if the two density operators commute, we can write $\rho=\sum_{k} p_{k}|k\rangle\langle k|$ and $\sigma=\sum_{k} q_{k}|k\rangle\langle k|$ for an orthonormal basis $\{|k\rangle\}$. Then, we have $F_{B}(\rho, \sigma)=$ $\sum_{k} \sqrt{p_{k} q_{k}}$ which is the classical fidelity $F\left(p_{k}, q_{k}\right)$ between the two probability distributions $p_{k}$ and $q_{k}$.

[35] This is the quantum version of the Kolmogorov distance $D\left(p_{k}, q_{k}\right):=\frac{1}{2} \sum_{k}\left|p_{k}-q_{k}\right|$ which is defined for two classical probability distributions $\left\{p_{k}\right\}$ and $\left\{q_{k}\right\}$.

[36] Roughly speaking, a trace-class operator is a linear operator for which we can define the trace. More rigorously, a bounded linear operator $O$ over a separable Hilbert space $\mathcal{H}$ is called "trace-class" if for some (and hence all) orthonormal bases $\left\{\left|\varphi_{k}\right\rangle\right\}$ we have $\sum_{k}\left\langle\varphi_{k}\left|\sqrt{O^{\dagger} O}\right| \varphi_{k}\right\rangle<$ $\infty$. In such a case, both the trace $\operatorname{Tr} O$ and the trace norm $\|O\|_{1}:=\operatorname{Tr} \sqrt{O^{\dagger} O}$ are correctly defined and finite. Furthermore, we can define a whole class of Schatten $p$ norms $\|O\|_{p}:=\left(\operatorname{Tr}|O|^{p}\right)^{1 / p}$ for every real $p \geq 1$. This class includes the trace norm for $p=1$ and the HilbertSchmidt norm for $p=2$.

[37] V. Kargin, Ann. Statist. 33, 959 (2005).

[38] With the notation $\left[\hat{\mathbf{x}}, \hat{\mathbf{x}}^{T}\right]$ we mean a matrix with entries $\left[\hat{\mathbf{x}}_{i}, \hat{\mathbf{x}}_{j}\right]$, where $i, j=1, \cdots, 2 n$. Analogously, $\left\{\hat{\mathbf{x}}, \hat{\mathbf{x}}^{T}\right\}$ is a matrix with entries $\left\{\hat{\mathbf{x}}_{i}, \hat{\mathbf{x}}_{j}\right\}$.

[39] R. Simon, N. Mukunda, and B. Dutta, Phys. Rev. A 49, 1567 (1994).
[40] J. Williamson, Am. J. Math. 58, 141 (1936).

[41] S. Pirandola, A. Serafini, and S. Lloyd, Phys. Rev. A 79, 052327 (2009).

[42] P. van Loock and S. L. Braunstein, Phys. Rev. A 61, 010302(R) (1999).

[43] N. Takei, H. Yonezawa, T. Aoki, and A. Furusawa, Phys. Rev. Lett. 94, 220502 (2005).

[44] S. Pirandola, D. Vitali, P. Tombesi, and S. Lloyd, Phys. Rev. Lett. 97, 150403 (2006).

[45] S.-H. Tan, B. I. Erkmen, V. Giovannetti, S. Guha, S. Lloyd, L. Maccone, S. Pirandola, and J. H. Shapiro, Phys. Rev. Lett. 101, 253601 (2008).

[46] S. Lloyd, Science 321, 1463 (2008).

[47] S. Pirandola, Phys. Rev. Lett. 106, 090504 (2011).

[48] S. Pirandola, C. Lupo, V. Giovannetti, S. Mancini, and S. L. Braunstein, New J. Phys. 13, 113012 (2011).

[49] R. Nair, Phys. Rev. A 84, 032312 (2011).

[50] O. Hirota, e-print arXiv:1108.4163 (2011).

[51] A. Bisio, M. Dall'Arno, and G. M. D'Ariano, Phys. Rev. A 84, 012310 (2011).

[52] M. Dall'Arno, A. Bisio, G. M. D'Ariano, M. Miková, M. Ježek, and M. Dušek, Phys. Rev. A 85, 012308 (2012).

[53] S. Guha , S.-H. Tan, and M. M. Wilde, "Explicit capacity-achieving receivers for optical communication and quantum reading," e-print arXiv:1202.0518 (2012). 